\title{
Solar X-rays from 0.3 A.U.: the ChemiX Bragg Spectrometer on Interhelioprobe
}

\author{
Janusz Sylwester ${ }^{1}$, Marek Siarkowski, Jarosław Bąkała, Żaneta \\ Szaforz, Mirosław Kowaliński, Marek Stęślicki, Barbara Sylwester, \\ Zbigniew Kordylewski, Oleksiy Dudnik ${ }^{2}$, Vladimir D. Kuznetsov ${ }^{3}$, \\ Valery Polansky ${ }^{3}$, Sergey Kuzin ${ }^{4}$ and Kenneth J. H. Phillips ${ }^{5}$ \\ ${ }^{1}$ Space Research Centre, Polish Academy of Sciences, Wrocław, Poland \\ email: js@cbk.pan.wroc.pl \\ ${ }^{2}$ Institute of Radio Astronomy, National Academy of Sciences of Ukraine, Kharkiv, Ukraine \\ email: dudnik@rian.kharkov.ua \\ ${ }^{3}$ Pushkov Institute of Terrestrial Magnetism, Ionosphere and Radiowave Propagation, RAS, \\ Moscow Troitsk, Russia \\ email: kvd@izmiran.ru \\ ${ }^{4}$ Laboratory of X-ray Astronomy of the Sun, P. N. Lebedev Physical Institute, RAS, Moscow, \\ Russia \\ email: kuzin@sci.lebedev.ru \\ ${ }^{5}$ Dept. of Earth Sciences, Natural History Museum, London SW7 5BD, U.K. \\ email: kennethjhphillips@yahoo.com
}

\begin{abstract}
ChemiX is a Bragg crystal spectrometer that will fly on the two Interhelioprobe spacecraft due for launch in 2025 and 2026. The spacecraft perihelion will be only 0.3 A.U. and the orbit inclination up to $30^{\circ}$, and so instruments on board will have a close view of solar active regions and flares and regions near each solar pole. The ChemiX X-ray spectrometer, built by a consortium of groups led by the Space Research Centre, Polish Academy of Sciences, will fly on each of the spacecraft, and observe X-ray spectra in the $1.5-9 \AA$ range. Spectral lines in this range include resonance lines of helium-like and hydrogen-like ions of elements such as $\mathrm{Fe}, \mathrm{Ca}$, $\mathrm{Ar}, \mathrm{S}$, and $\mathrm{Si}$, with less abundant elements such as $\mathrm{K}$ and $\mathrm{Cl}$ represented by weaker lines which the high sensitivity of ChemiX should be able to detect. The free-free and free-bound continua should also be detected since instrumental background will be eliminated. Three of the seven channels of ChemiX will be in a "dopplerometer" arrangement by which spatial and spectral shifts present in flare impulsive stages can be disentangled.
\end{abstract}

Keywords. techniques: spectroscopic, Sun: abundances, Sun: corona, Sun: flares, Sun: X-rays, gamma rays

\section{Introduction}

X-ray emission is formed in optically thin multi-million Kelvin solar plasmas such as are present in the quiet Sun, active regions, or flares. X-ray spectral lines are emitted by these plasmas and are due to transitions in highly ionized elements such as $\mathrm{Mg}, \mathrm{Al}, \mathrm{Si}, \mathrm{S}$, $\mathrm{Ar}, \mathrm{Ca}, \mathrm{Fe}$, and $\mathrm{Ni}$, with weaker line emission due to less abundant elements such as $\mathrm{K}$ and $\mathrm{Cl}$. Many of the principal emission lines, which are mainly due to transitions in hydrogenlike and helium-like ions of elements, fall in the spectral range $1.5-9 \AA$. There is also a prominent continuum formed of free-free (bremsstrahlung) and free-bound radiation; free-bound emission depends on the plasma composition, with abundant elements being the most important emitters. 


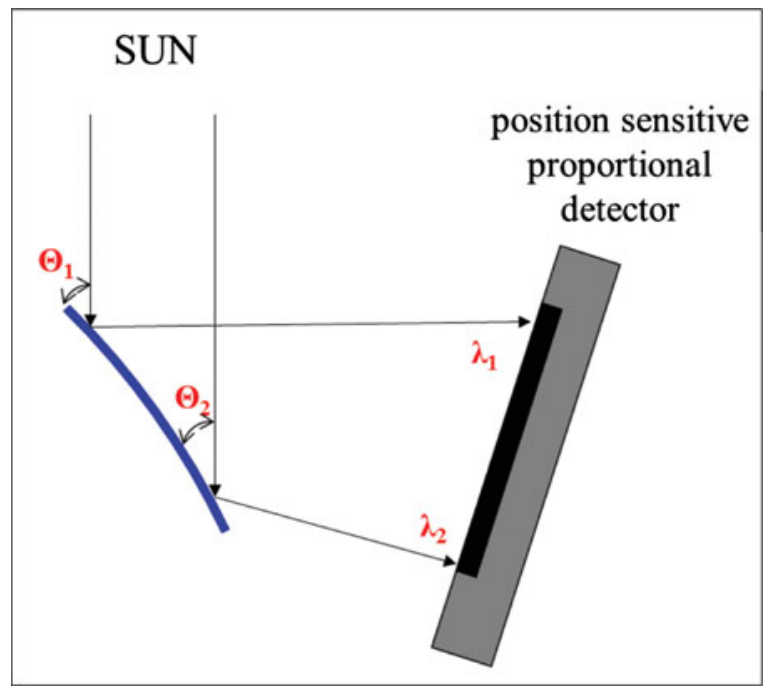

Figure 1. Ray diagram for bent crystal spectrometer. X-ray spectrum is instantaneously recorded by the position-sensitive detector within the wavelength range between $\lambda_{1}$ and $\lambda_{2}$.

Spectrometers observing this $\mathrm{X}$-ray range are able to obtain information relating to the elemental composition of solar plasma, plasma flows and turbulence, measurement of temperature (for isothermal plasmas) or the distribution of plasma with temperature, and the presence of non-thermal electrons. The instrumentation should have several requirements: these include fine spectral resolution, good sensitivity, high time resolution, and the ability to detect the continuum level. The spectral resolution should be adequate to observe lines of particular elements, and the instrument's sensitivity be large enough for lines of lower-abundance elements to be seen. The continuum is often confused with instrumental background, in particularly Bragg crystal spectrometers as there is often fluorescence from the crystal material. This was a problem in spectrometers on the Intercosmos, Pr8-1, Hinotori spacecraft; other examples are most of the channels of the X-ray Polychromator (XRP) on Solar Maximum Mission (operational in the 1980s: Acton et al. 1980) and the Bragg Crystal Spectrometer on Yohkoh (operational in the 1990s: Culhane et al. 1991). A careful adjustment of instrument parameters during the operational period of the RESIK instrument on the CORONAS-F spacecraft (operational 2001-2003: Sylwester et al. 2005) allowed the elimination of this background for two of the four channels, so an improvement in the spectral quality was achieved.

In this contribution, we describe the ChemiX spectrometer that will be included in the payload of the Russian Interhelioprobe interplanetary spacecraft that are due for launch in 2025 and 2026 in orbits that will take them to the near vicinity of the Sun.

\section{Bent Crystal spectrometers}

X-ray crystal spectrometers have generally obtained spectra by rotating flat crystals, so that spectra may be built up by varying the angle of incidence $\theta$ in the Bragg condition $\lambda=2 d \sin \theta$ ( $\lambda=$ wavelength, $d=$ crystal lattice spacing). A considerable advantage may be obtained by the use of bent crystals so that the angle of incidence is slightly different for different points across the crystal face: see Figure 1. In this figure, rays from a solar source such as an active region or flare are incident from the top on to a bent crystal with incident angles $\theta_{1}$ and $\theta_{2}$ at different positions on the crystal giving rise to diffracted 


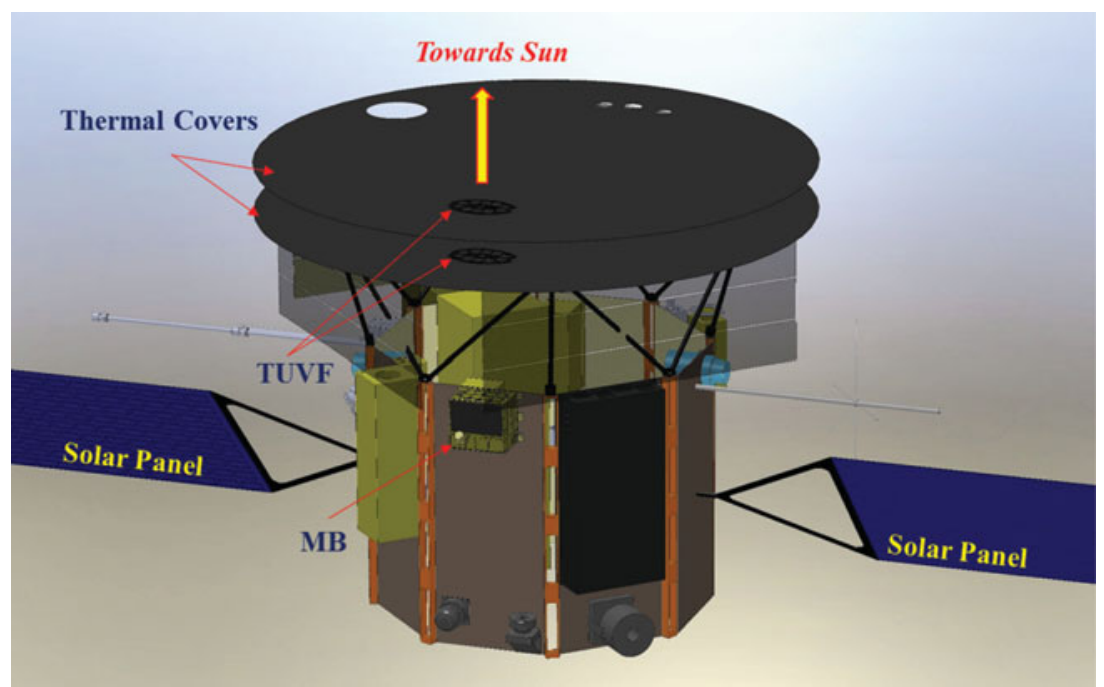

Figure 2. Interhelioprobe spacecraft showing location of ChemiX (MB, Measurement Block). Solar radiation is incident from the top on to two thermal covers. A thermal UV filter (TUVF) allows only X-ray emission to be incident on ChemiX. Other instruments on board will be a pin-hole soft X-ray camera $(0.5-10 \mathrm{keV})$ to provide context spatial information and a unit to detect particles.

rays of slightly different wavelengths $\lambda_{1}$ and $\lambda_{2}$ which are sensed by a position-sensitive proportional counter having a beryllium window.

Among the advantages in the use of bent crystal spectrometers is the study of dynamic phenomena such as solar flares. Thus, spectra may be instantaneously formed over a short time interval (the data-gathering interval), typically a few seconds, by the read-out of the detector. This is necessary for following the impulsive stages of flares which may last much less than a minute. Secondly, the sensitivity of the instrument is generally much larger than for a scanning flat crystal spectrometer, which allows weaker spectral lines such as the resonance lines of low-abundance elements to be observed. Thirdly, there are no (or at most very few) moving parts in bent crystal spectrometers, thus reducing the possibility of mechanical failures as occurred with some of the spacecraft instrumentation mentioned above.

\section{ChemiX on Interhelioprobe}

Two Russian spacecraft, Interhelioprobe-1 and Interhelioprobe-2, will be launched in 2025 and 2026 in orbits that will take them to about 0.3 A.U. from the Sun with inclinations of $30^{\circ}$ so that regions around each solar pole can be viewed. See Figure 2 for spacecraft details. The spacecraft front face will have thermal covers to protect the instrumentation on board from the greatly increased solar radiation at perihelion (approximately an order of magnitude greater than at the distance of the Earth). A thermal ultraviolet filter (TUVF in Figure 2) blocks all but X-ray emission being incident on the Measurement Block (MB) of ChemiX.

ChemiX will have a total of seven channels. Four of these will cover X-ray spectra in the $1.5-8.8 \AA$ range, each channel's range slightly overlapping. Table 1 gives details of the crystals and wavelength ranges of the channels. Channels 1-4 are spectrometer channels with one crystal per channel. The crystals are bent to radii of between 166 and $410 \mathrm{~mm}$, much smaller than the radii of crystals included in the RESIK or SMM BCS instruments. 
Table 1. ChemiX channels: crystals and detectors

\begin{tabular}{llcccl}
\hline $\begin{array}{l}\text { Chan. } \\
\text { No. }\end{array}$ & $\begin{array}{l}\text { Crystal and } \\
\text { diff. plane }\end{array}$ & $\begin{array}{c}2 d \\
(\AA)\end{array}$ & $\begin{array}{l}\text { Wavelength } \\
\text { range }(\AA)\end{array}$ & $\begin{array}{l}\text { Spectr. resol. } \\
\mathrm{m \AA} \mathrm{per} \mathrm{pixel}\end{array}$ & $\begin{array}{l}\text { Curvature } \\
\mathrm{R}(\mathrm{mm})\end{array}$ \\
\hline 1 & Si 111 & 6.27 & $1.50-2.71$ & 1.4 & 176.0 \\
2 & Quartz 10̄10 & 8.51 & $2.70-4.30$ & 1.8 & 166.7 \\
3 & KDP 011 & 10.19 & $4.29-5.22$ & 0.98 & 410.4 \\
4 & KAP 001 & 26.64 & $5.20-8.80$ & 4.2 & 364.7 \\
\hline 5 & LiF 022 & 2.85 & $1.84-1.95$ & 0.12 & 650.0 \\
6 & Si 111 & 6.27 & $3.15-3.32$ & 0.11 & 2500.0 \\
7 & Si 111 & 6.27 & $3.90-4.08$ & 0.19 & 1000.0 \\
\hline
\end{tabular}

Channels 5-7 are "dopplerometer" channels, i.e. the crystals arranged in such a way that spatial shifts cause displacements in one direction along the crystal but spectral shifts displacements in the opposite directions. By this means spatial and spectral shifts, which are commonly present at the outset of solar flares, can be distinguished. The principle is described by Sylwester et al. (2015). The spectral ranges for these channels include the lines of Fe XXV, Ca XIX, and Ar XVII, i.e. helium-like ions of abundant elements with lines emitted at temperatures between $4 \mathrm{MK}$ (characteristic of dynamic active regions) and $>20 \mathrm{MK}$ (characteristic of solar flares). It is estimated that the fluorescence from the crystals in each channel should be negligible. A considerable advance over earlier X-ray spectrometers will be the use of back-illuminated CCDs, manufactured by the e2v company, as the detectors. These CCDs will have a $1024 \times 256$ pixel format and $26 \mu \mathrm{m}$ square pixels. Examples of simulated spectra from spectrometer channel 2 and dopplerometer channel 3 are shown on Figure 3. It is seen that the spectral resolution in the dopplerometer channels (of the order of $10^{-5} \AA$ ) is good enough to observe physical line profiles which can be affected by:

- presence of radial component of bulk plasma motions

- presence of microturbulence in the plasma

Among the subsystems of ChemiX will be a particle detector (provided by the Institute of Radio Astronomy, Ukrainian Academy of Sciences) which will issue a "switch off" flag to ChemiX in case of increased particle emission to levels injurious to the instrument's health and also obtain particle spectra. It will provide estimates of background count rates of the ChemiX CCDs. A pin-hole CCD camera with 1 arcminute resolution will provide spatial context images so that active regions can be identified in the event of a flare. There will be a target pointing platform which will carry all the crystals of ChemiX and the CCD detectors and which will lock on to a target in $<1 \mathrm{~s}$. There will also be a "spectral atlas spectrometer" which will record X-ray spectra in the $1.5-9 \AA$ range within a selected field of view, with a variable data gathering interval to maintain a total of 10000 photon counts in a spectrum. This will enable spectral line and continuum fluxes to be obtained with great accuracy, approximately twice that of the RESIK instrument, when the instrument is at perihelion. Finally, the dopplerometer channels of ChemiX will also give information on spectral line shapes so that upflows or other plasma motions which occur at the flare impulsive stage can be identified.

\section{Summary and Conclusions}

The launch of the Interhelioprobe spacecraft will mark a new era in solar physics, with close-up views of the Sun including the polar regions. The ChemiX instrument which will be included will obtain X-ray spectra in seven channels covering the $1.5-9 \AA$ spectral 

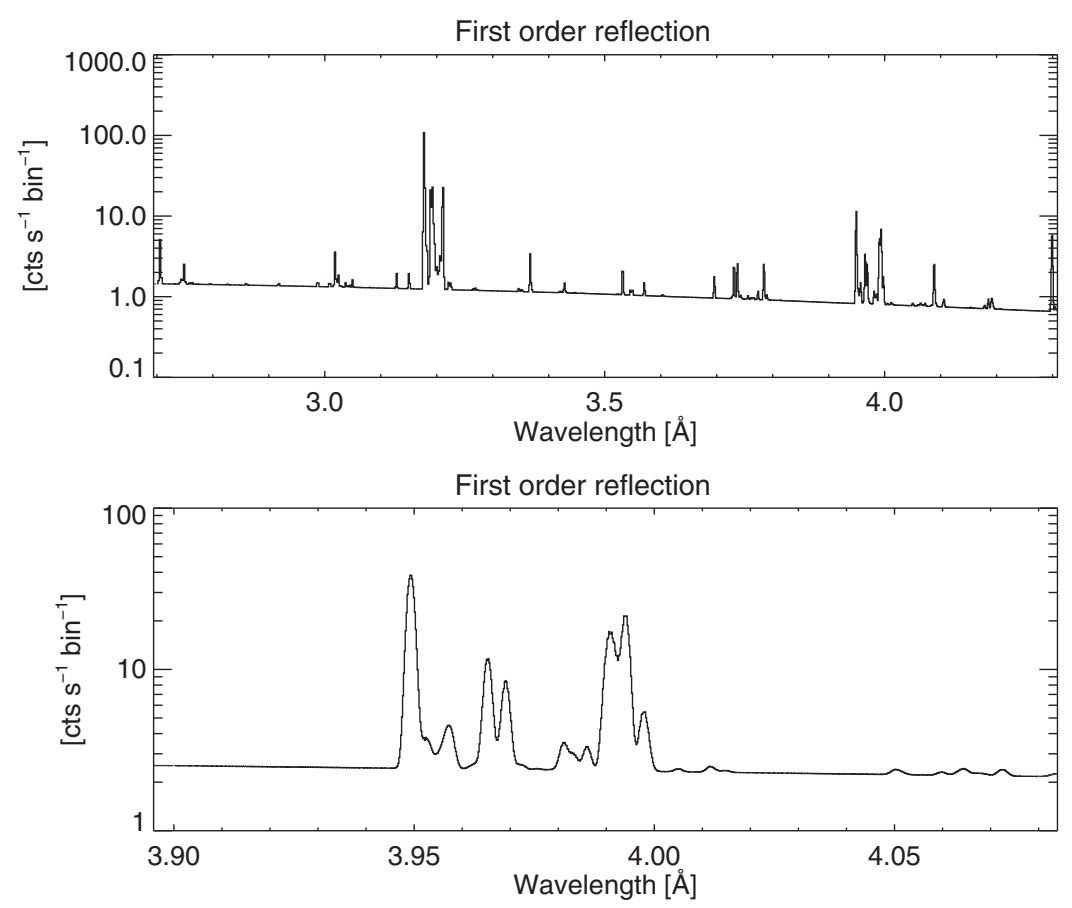

Figure 3. Spectra simulated using the CHIANTI database for a typical GOES M5 solar flare as seen by ChemiX on the CCD at the spacecraft distance 0.3 A.U. from the Sun. Top panel: spectrometer channel 2 with Ca XIX and Ar XVII He-like triplet and dielectronic satellites clearly seen. Bottom panel: Dopplerometer channel 3 with highly ionized Ar XVII line profiles resolved.

range. Four of the channels will be spectrometer channels and three dopplerometer channels that will provide information about the spatial and spectral shifts that have been observed during the impulsive stages of solar flares. As such, ChemiX should be a large step forward from the technology of the X-ray spectrometers on board spacecraft during the 1980 s and up to 2003 on the CORONAS-F spacecraft.

\section{Acknowledgements}

We acknowledge the support from the Polish National Science Centre grants: UMO2011/01/M/ST9/05878, UMO-2011/01/M/ST9/06096, UMO-2013-11/B/ST9/00234 and the support from Ukrainian Academy of Sciences grants: 2013/1.57.11 and 2014/1.57.1.11 2015/1.57.2.16. We also acknowledge use of the CHIANTI atomic database and code for calculating spectra presented in Figure 3. CHIANTI is a collaborative project involving George Mason University, University of Michigan (USA) and University of Cambridge (UK). LPI was supported by program \#7 of Presidium of Russian Academy of Science.

\section{References}

Acton, L. W., et al. 1980, Sol. Phys., 65, 53

Culhane, J. L., et al. 1991, Sol. Phys., 136, 89

Sylwester, J., et al. 2005, Sol. Phys., 226, 45

Sylwester, J., et al. 2015, Sol. Phys., 290, 3683 